Archive for

Organic Chemistry

Arkivoc 2019, part v, 89-95

\title{
Transformations of perfluorotoluene by the action of 2-mercaptoethanol
}

\author{
Borislav V. Koshcheev, Roman A. Bredikhin,* Alexander M. Maksimov, Vyacheslav E. Platonov, \\ and Vladimir V. Shelkovnikov
}

N.N. Vorozhtsov Novosibirsk Institute of Organic Chemistry of the Siberian Branch of Russian Academy of Sciences, 630090 Novosibirsk, Russian Federation

Email: scisecretary@nioch.nsc.ru

\section{Dedicated to the memory of academician D.G. Knorre}

Received 09-10-2018

Accepted 12-14-2018

Published on line 02-19-2019

\section{Abstract}

The reaction of perfluorotoluene and 2-mercaptoethanol in the presence of $\mathrm{K}_{2} \mathrm{CO}_{3}$ in $\mathrm{DMF}$ has been studied and experimental procedures for selective introduction of one, two and three ethanolthiyl groups into perfluorotoluene have been elaborated.

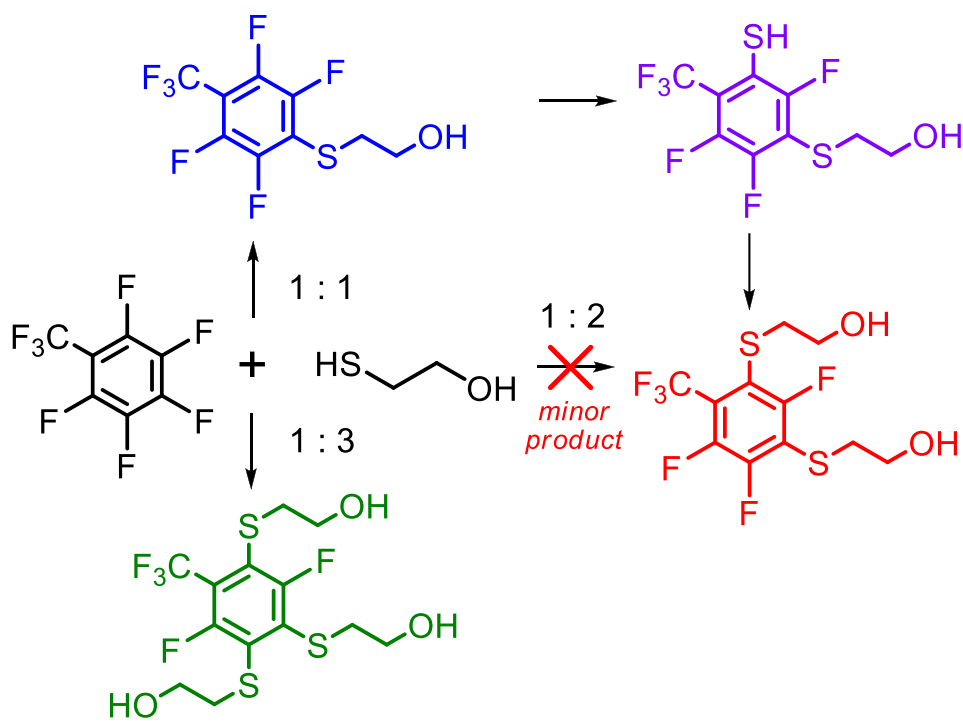

Keywords: Perfluorotoluene, 2-mercaptoethanol, aromatic nucleophilic substitution, regioselectivity 


\section{Introduction}

For the last few decades, intensive investigations have been devoted to materials that demonstrate non-linear optical (NLO) properties and various strategies for the synthesis of dendrimer materials with NLO properties have been described. ${ }^{1-3}$ Moreover, some results provide evidence that the correct choice of 'a suitable isolation group' might have a strong influence on the macroscopic NLO properties in polymers. ${ }^{4}$ For example, the introduction of polyfluoroarenes as isolation groups improves the stability and enhances the second harmonic coefficient $\mathrm{d}_{33}{ }^{5,6} \mathrm{It}$ was also shown that tetrafluorophenylene-linkages compared to non-fluorinated ones provided higher quantum yields of fluorescence, but with smaller two-photon absorptivity. ${ }^{7}$ The use of fluorinated species can also aid in the formation of non-covalent interactions that improve the organization of the molecules in the crystal phase and thus the electrooptic properties. ${ }^{4,8}$ For these reasons, a notable number of studies were devoted to the synthesis of polyfluorinated arylenes. ${ }^{9-12}$ Recently, a versatile method for construction of fluorinated poly(aryl thioethers) was developed based on organo-catalyzed nucleophilic aromatic substitution of silyl-protected dithiols. ${ }^{13}$

The current work investigates the transformations of perfluorotoluene under the action of 2-mercaptoethanol and has been aimed at the synthesis of substituted derivatives of perfluorotoluene as potential blocks for dendrimer construction. The study focused on the regioselectivity of the reaction and elaboration of reliable synthetic procedures. 2-Mercaptoethanol was selected because its hydroxyl groups enable the further functionalization of the products while the sulfur can be oxidized to either the sulfoxide or sulfone state. Examples of the reactions of polyfluoroarenes with excess of S-nucleophiles are known. ${ }^{14-16}$

\section{Results and Discussion}

The reaction of perfluorotoluene (1) with an equimolar amount of 2-mercaptoethanol (2) in the presence of potassium carbonate afforded 2-\{[2,3,5,6-tetrafluoro-4-(trifluoromethyl)phenyl]thio\}ethan-1-ol (3). Potassium carbonate was used to transform thiol 2 to an even more nucleophilic thiolate-anion. The direction of aromatic nucleophilic fluorine substitution was typical for the reactions of subsituted pentafluorophenyls $\left(\mathrm{C}_{6} \mathrm{~F}_{5} \mathrm{X}\right)$ with various nucleophiles. ${ }^{17}$ Recently, kinetics for the nucleophilic aromatic monosubstitution of parafluorine by the action of thiol $\mathbf{2}$ in aq. MeCN were measured for a series of polyfluoroarenes $\mathrm{C}_{6} \mathrm{~F}_{5} \mathrm{X}$, including compound $1 .^{18}$ Compound $\mathbf{3}$ was also obtained by alkylation of 2,3,5,6-tetrafluoro-4-trifluoromethylbenzenethiol (4) by 2-chloroethanol (5) in aqueous alkali.

When arene 1 reacted with thiol 2 (2 equiv), a mixture of mono- (3), di- (6) and trisubstituted (7) perfluorotoluenes in the ratio $43: 17: 40\left({ }^{19} \mathrm{~F} \mathrm{NMR}\right)$ was formed, while the action of 3 equivalents of thiol 2 to compound $\mathbf{1}$ afforded exclusively trisubstituted compound $\mathbf{7}(70 \%)$ in a one-pot procedure. On the one hand, the nucleophilic substitution of fluorine at the C3 position of compound $\mathbf{3}$ was well known and the regioselectivity was attributed to the directing effects of the electron-withdrawing trifluoromethyl and electron-donating alkanethiyl groups. On the other hand, the introduction of one more alkanethiyl group assists the subsequent nucleophilic substitution of fluorine in the para-position to it $\left(6,{ }^{6} \mathrm{~F}\right)$ with formation of compound $7 .^{16}$ The analogous reaction pathway has been observed in reactions of other polyfluoroarenes. For example, the reaction of 1,2,4,5-tetrafluorobenzene with sodium tert-butylthiolate gave 1,4-bis(tertbutylthio)-2,5-difluorobenzene in $87 \%$ yield. ${ }^{19}$ Transformations of hexafluorobenzene under the action of sodium isopropylthiolate or cesium propylthiolate led to the formation of 1,4-bis(isopropylthio)-2,3,5,6tetrafluorobenzene and 1,4-bis(propylthio)-2,3,5,6-tetrafluorobenzene in 71 and $81 \%$ yields, respectively. ${ }^{19,20}$ 
Furthermore, the reaction of a 1:1 ratio of hexafluorobenzene with 2-mercaptoethanol (2) or sodium 2hydroxyethanethiolate in liquid ammonia gave exclusively the 2,2'-(perfluoro-1,4-phenylene)bis(sulfanediyl)diethanol in $\sim 100 \%$ yield ${ }^{21}$ which was consistent with our results. The observed results were attributed to the greater polarizability of the sulfur than fluorine atom. ${ }^{22}$ A structure specific synthesis of the disubstituted product 6 was achieved from compound 3 in 2 steps: arene 3 was reacted with potassium hydrosulfide to afford thiol 8, which was subsequently treated with 2-chloroethanol (5) (Scheme 1).

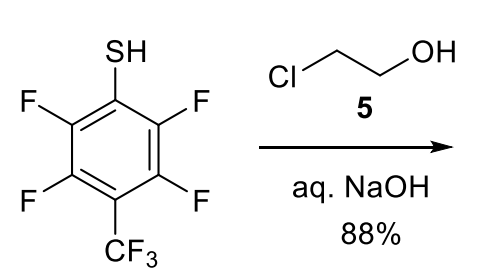

4<smiles>CCCSc1c(F)c(F)c(C(F)(F)F)c(F)c1F</smiles>

3<smiles>OCCSc1c(F)c(F)c(C(F)(F)F)c(S)c1F</smiles>

8
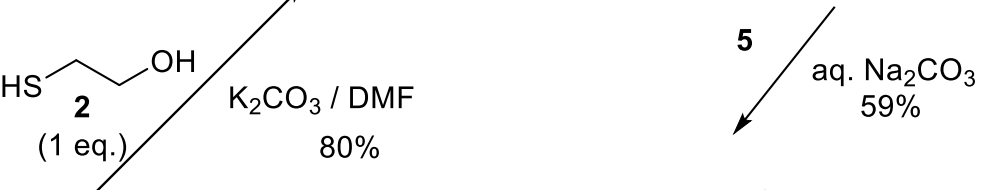

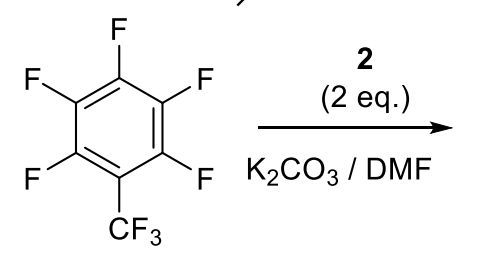

1

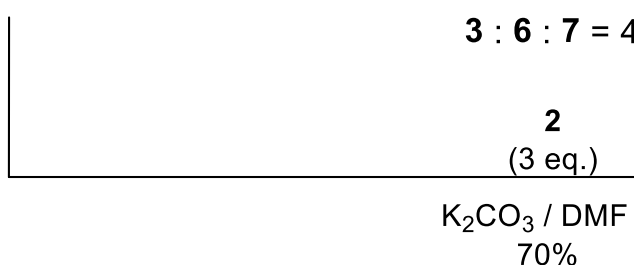

(c)

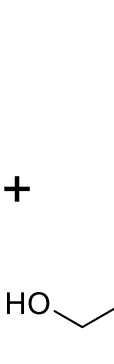




\section{Experimental Section}

General. Analytical measurements. The NMR spectra of reaction mixtures or individual compounds were recorded on Bruker AVANCE 300 [300.13 $\left.\left({ }^{1} \mathrm{H}\right), 282.4\left({ }^{19} \mathrm{~F}\right) \mathrm{MHz}\right]$, Bruker AV-400 [400.13 $\left.\left({ }^{1} \mathrm{H}\right), 100.6\left({ }^{13} \mathrm{C}\right) \mathrm{MHz}\right]$ or Bruker DRX-500 [500.13 $\left({ }^{1} \mathrm{H}\right), 125.76\left({ }^{13} \mathrm{C}\right) \mathrm{MHz}$ ] spectrometers for solutions of samples in $\mathrm{CCl}_{4},\left(\mathrm{CD}_{3}\right)_{2} \mathrm{CO}_{2}$ or $\mathrm{CD}_{3} \mathrm{CN}$ [for ${ }^{1} \mathrm{H}$ and ${ }^{19} \mathrm{~F}$ ], $\mathrm{CDCl}_{3}$ [for ${ }^{1} \mathrm{H},{ }^{13} \mathrm{C}$ and ${ }^{19} \mathrm{~F}$ ] or $\mathrm{CD}_{3} \mathrm{CN}$ [for ${ }^{13} \mathrm{C}$ and ${ }^{19} \mathrm{~F}$ ]. NMR coupling constants $(\mathrm{J})$ were measured in Hertz $(\mathrm{Hz})$. IR spectra were recorded on a spectrophotometer Bruker Vector 22 from pellets with $\mathrm{KBr}$ for solid and from films for liquid samples. UV spectra were obtained on a spectrophotometer Hewlett Packard 8453 from solutions in ethanol. The molecular mass and elemental composition was determined from the high resolution mass spectra taken on Thermo Electron Corporation DFS instrument (ionizing electrons energy $70 \mathrm{eV}$ ). Elemental analysis was performed on an EURO EA 3000 automatic CHNS analyzer. ${ }^{23}$ Fluorine determination was performed spectrophotometrically using a Cary-50 spectrophotometer. GC-MS spectra were measured on Hewlett-Packard G1081A instrument equipped with a gas chromatograph HP 5890 Series II and a mass-selective detector HP 5971 (EI, $70 \mathrm{eV}$ ), capillary column HP-5 (5\% of diphenyl-, 95\% dimethylsiloxane) $30 \mathrm{~m} \times 0.25 \mathrm{~mm} \times 0.25 \mu \mathrm{m}$, carrier gas helium, flow rate $1 \mathrm{~mL} / \mathrm{min}$. Injector temperature $280{ }^{\circ} \mathrm{C}$, ion source temperature $173{ }^{\circ} \mathrm{C}$. Scanning rate $1.2 \mathrm{scan} / \mathrm{s}$ in mass region 30-650 a.u.m. Analytic GLC was carried out on a chromatograph Hewlett Packard 5980, equipped with a quartz capillary column HP-5 (stationary phase dimethyl diphenyl polysiloxane block copolymer), $30 \mathrm{~m} \times 0.52 \mathrm{~mm} \times 2.6 \mu \mathrm{m}$, and a thermal conductivity detector (TCD). The melting points were measured on a Koeffler heating block and are uncorrected.

All starting compounds are widely used commercially available products of reagent grade and purified in the usual manner whenever necessary prior to use. Perfluorotoluene $(\mathbf{1}, 97 \%)$ was obtained from P\&M Invest, and 2,3,5,6-tetrafluoro-4-(trifluoromethyl)benzenethiol (4) was obtained from compound 1 according to described procedure. ${ }^{24}$

2-\{[2,3,5,6-Tetrafluoro-4-(trifluoromethyl)phenyl]thio\}ethan-1-ol (3). Procedure A. Reaction of perfluorotoluene (1) and 2-mercaptoethanol (2) in ratio 1:1. Compound 2 (11.44 g, $145.96 \mathrm{mmol})$ was added to the solution of arene $1(33.82 \mathrm{~g}, 141.84 \mathrm{mmol})$ in DMF $(290 \mathrm{~mL})$ in the presence of $\mathrm{K}_{2} \mathrm{CO}_{3}(21.24 \mathrm{~g}, 152.14$ $\mathrm{mmol})$. After $4.5 \mathrm{~h}$ stirring at room temperature the resulted mixture was poured into $5 \%$ aq. $\mathrm{HCl}(500 \mathrm{~mL})$, the organic layer was washed with water $(2 \times 250 \mathrm{~mL})$ and dried $\left(\mathrm{MgSO}_{4}\right)$. The crude product $(35.28 \mathrm{~g}, 95 \% \mathrm{GLC}$, $80 \%$ yield) was purified by vacuum distillation.

Procedure B. Alkylation of 2,3,5,6-tetrafluoro-4-(trifluoromethyl)benzenethiol (4) by 2-chloroethanol (5). Arenethiol 4 (4.54 g, $18.2 \mathrm{mmol}$ ) was dissolved in $1 \mathrm{M} \mathrm{NaOH}(20 \mathrm{~mL})$, then compound 5 (3.54 g, $44.0 \mathrm{mmol})$ was added. After stirring overnight at room temperature, the mixture was extracted with $\mathrm{CHCl}_{3}(2 \times 10 \mathrm{~mL})$, washed with water $(2 \times 50 \mathrm{~mL})$, dried $\left(\mathrm{MgSO}_{4}\right)$ and the solvent was evaporated to give compound 3 (4.69 $\mathrm{g}$, $88 \%$ ) as a colorless oil, $\mathrm{mp} 16-17{ }^{\circ} \mathrm{C}$. bp $83-85{ }^{\circ} \mathrm{C}\left(2\right.$ Torr). IR (neat, $\left.v_{\max } \mathrm{cm}^{-1}\right)$ 3360(w), 2945(w), 2884(w), 1647(m), 1479(s), 1398(w), 1329(s), 1182(m), 1148(s), 1063(w), 980(s), 831(m), 716(m). UV, $\lambda_{\max }, \mathrm{nm}(\log \varepsilon)$ : 209 (3.87), 279 (3.81). ${ }^{1} \mathrm{H}$ NMR $\left(\mathrm{CDCl}_{3}, 500.13 \mathrm{MHz}\right): \delta_{\mathrm{H}} 2.66$ (br.s, $\left.1 \mathrm{H}, \mathrm{OH}\right), 3.18\left(\mathrm{t},{ }^{3} \mathrm{JHH}_{\mathrm{HH}} 5.9,2 \mathrm{H}, \mathrm{CH}_{2} \mathrm{~S}\right), 3.75(\mathrm{t}$, $\left.{ }^{3} J_{\mathrm{HH}} 5.9,2 \mathrm{H}, \mathrm{CH}_{2} \mathrm{O}\right) .{ }^{13} \mathrm{C} \mathrm{NMR}\left(\mathrm{CDCl}_{3}, 125.76 \mathrm{MHz}\right), \delta_{\mathrm{C}} 37.2\left(\mathrm{t},{ }^{4} J_{\mathrm{CF}} 3.2, \mathrm{SCH}_{2}\right), 61.4\left(\mathrm{OCH}_{2}\right), 109.2\left(\mathrm{qt},{ }^{2} J_{\mathrm{CF}} 35.1\right.$, $\left.{ }^{2} J_{C F} 12.9, \underline{C}_{A r C F}\right), 119.8\left(t,{ }^{2} J_{C F} 19.9, \underline{C} \underline{A r}_{A}\right), 121.0\left(q,{ }^{1} J_{C F} 228.9, \underline{C F}_{3}\right), 143.0-145.4\left(m, \underline{C}_{A r} F\right), 146.0-148.2\left(m, \underline{C}_{A r} F\right)$. ${ }^{19} \mathrm{~F} \mathrm{NMR}\left[\mathrm{CDCl}_{3}, 282.4 \mathrm{MHz}\right]: \delta_{\mathrm{F}}-141.5--141.1[\mathrm{~m}, 2 \mathrm{~F}, \mathrm{~F}-(2,6)],-133.5--133.3[\mathrm{~m}, 2 \mathrm{~F}, \mathrm{~F}-(3,5)],-57.7\left(\mathrm{t},{ }^{4} J_{\mathrm{FF}}\right.$ 21.7, 3F, $\mathrm{CF}_{3}$ ). Anal. calcd for $\mathrm{C}_{9} \mathrm{H}_{5} \mathrm{~F}_{7} \mathrm{OS}, \%$ : C 36.75; H 1.71; F 45.21; S 10.90; $\mathrm{m} / \mathrm{z} 293.9944$. Found, \%: C 36.76; H 1.83; F 45.24; S 10.85; $m / z 293.9937$. 
2,2'-\{[2,4,5-Trifluoro-6-(trifluoromethyl)-1,3-phenylene]bis(sulfanediyl)\}diethanol

(6).

Reaction of perfluorotoluene (1) and 2-mercaptoethanol (2) in ratio 1:2. Compound 2 (0.88 g, $11.3 \mathrm{mmol})$ was added to the solution of arene $1(1.32 \mathrm{~g}, 5.59 \mathrm{mmol})$ in DMF $(5 \mathrm{~mL})$ in the presence of $\mathrm{K}_{2} \mathrm{CO}_{3}(1.54 \mathrm{~g}, 11.2 \mathrm{mmol})$. After $24 \mathrm{~h}$ stirring at $20-25{ }^{\circ} \mathrm{C}$ the resulted mixture was acidified with aq. $\mathrm{HCl}$ and subjected to analysis by means of ${ }^{19} \mathrm{~F}$ NMR, which revealed compounds 3,6 and 7 in ratio $43: 17: 40\left({ }^{19} \mathrm{~F} \mathrm{NMR}\right)$.

2-\{[2,3,6-Trifluoro-5-mercapto-4-(trifluoromethyl)phenyl]thio\}ethanol (8). A solution of potassium hydrosulfide in 1,2-ethanediol ( $4 \mathrm{M}, 41 \mathrm{~mL}, \mathrm{ca} .164 \mathrm{mmol}$ ) was prepared according to a previously described procedure ${ }^{24}$ and was added dropwise to a solution of compound 3 ( $\left.25.7 \mathrm{~g}, 98 \%, 85.61 \mathrm{mmol}\right)$ in DMF (155 $\left.\mathrm{mL}\right)$ for $35 \mathrm{~min}$ at $24-28{ }^{\circ} \mathrm{C}$ and the reaction mixture was stirred for $2 \mathrm{~h}$ at $28-23{ }^{\circ} \mathrm{C}$, poured into $15 \%$ aq. $\mathrm{HCl}(300$ $\mathrm{mL})$ and extracted with $\mathrm{CH}_{2} \mathrm{Cl}_{2}(2 \times 20 \mathrm{~mL})$. The extract was washed with water $(3 \times 50 \mathrm{~mL})$ and dried $\left(\mathrm{MgSO}_{4}\right)$. The solvent was distilled off, and the residue was crystallized to give thiol $8(16.64 \mathrm{~g}, 63 \%)$ as a colorless solid,

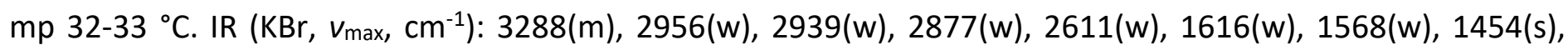
1412(m), 1356(s), 1279(s), 1194(m), 1173(s), 1142(s), 1128(s), 1068(s), 1014(m), 964(w), 947(m), 866(s), 773(m), 742(w), 723(m), 696(m), 665(w), 644(w). UV, $\lambda_{\max }, \mathrm{nm}(\log \varepsilon): 210$ (4.25), 240 (3.97), 274 (3.98), 353 (3.18). ${ }^{1} \mathrm{H} \mathrm{NMR}\left(\mathrm{CDCl}_{3}, 500.13 \mathrm{MHz}\right): \delta_{\mathrm{H}} 1.78$ (br.s, $\left.1 \mathrm{H}, \mathrm{OH}\right), 3.14\left(\mathrm{t}, 3^{3} \mathrm{JHH}_{\mathrm{H}} 6.0,2 \mathrm{H}, \mathrm{CH}_{2} \mathrm{~S}\right), 3.71\left(\mathrm{t},{ }^{3} \mathrm{~J}_{\mathrm{HH}} 6.0,2 \mathrm{H}\right.$,

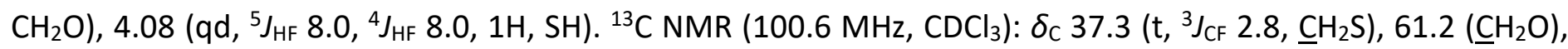
116.6 (qdd, $\left.{ }^{2} J_{C F} 33.2,{ }^{2} J_{C F} 10.6,{ }^{3} J_{C F} 2.0, \underline{C C F}_{3}\right), 117.2$ [dd, ${ }^{2} J_{C F} 26.4,{ }^{3} J_{C F} 4.4, \underline{C}-(5) S$ ], $117.8\left[d d,{ }^{2} J_{C F} 27.5,{ }^{2} J_{C F} 19.0\right.$, $\underline{\mathrm{C}}$-(1)S], $122.2\left(\mathrm{qd},{ }^{1} J_{\mathrm{CF}} 276.0,{ }^{3} J_{\mathrm{CF}} 1.3, \underline{\mathrm{CF}} 3\right.$ ), 146.2 [ddq, ${ }^{1} J_{\mathrm{CF}} 261.2,{ }^{2} J_{\mathrm{CF}} 16.9,{ }^{3} J_{\mathrm{CF}} 1.8, \underline{\mathrm{C}}-(3) \mathrm{F}$ ], $148.5\left[\mathrm{ddd},{ }^{1} J_{\mathrm{CF}}\right.$ $\left.247.8,{ }^{2} J_{\mathrm{CF}} 14.9,{ }^{3} J_{\mathrm{CF}} 5.7, \underline{\mathrm{C}}-(2) \mathrm{F}\right], 152.9$ [ddd, $\left.{ }^{1} J_{\mathrm{CF}} 237.9,{ }^{3} J_{\mathrm{CF}} 3.5,{ }^{4} J_{\mathrm{CF}} 3.5, \underline{\mathrm{C}}-(6) \mathrm{F}\right] .{ }^{19} \mathrm{~F} \mathrm{NMR}\left(\mathrm{CDCl}_{3}, 282.4 \mathrm{MHz}\right), \delta_{\mathrm{F}}$ -139.9 [qdd, ${ }^{4} J_{\mathrm{FF}} 26.4,{ }^{4} J_{\mathrm{FF}} 21.9,{ }^{5} J_{\mathrm{FF}} 13.6, \mathrm{~F}-(3)$ ], -131.6 [dd, ${ }^{3} J_{\mathrm{FF}} 21.9,{ }^{4} J_{\mathrm{FF}} 2.0, \mathrm{~F}-(2)$ ], $-102.6\left[\mathrm{dddq},{ }^{5} J_{\mathrm{FF}} 13.6,{ }^{4} J_{\mathrm{FH}}\right.$ 8.0, $\left.{ }^{4} J_{\mathrm{FF}} 2.0,{ }^{5} \mathrm{JFF}_{\mathrm{FF}} 2.0, \mathrm{~F}-(6)\right],-57.6$ (ddd, ${ }^{4} J_{\mathrm{FF}} 26.4,{ }^{5} J_{\mathrm{FH}} 8.5,{ }^{5} \mathrm{JFF}_{2.0}, 3 \mathrm{~F}, \mathrm{CF}_{3}$ ). Anal. calcd. for $\mathrm{C}_{9} \mathrm{H}_{6} \mathrm{~F}_{6} \mathrm{OS}_{2}: \mathrm{C} 35.07 ; \mathrm{H}$ 1.96; F 36.98; S 20.80; $\mathrm{m} / \mathrm{z}$ 307.9759. Found: C 35.38; H 2.08; F 37.01; S 21.03; $\mathrm{m} / \mathrm{z} 307.9755$.

2,2'-\{[2,4,5-Trifluoro-6-(trifluoromethyl)-1,3-phenylene]bis(sulfanediyl)\}diethanol (6). Alkylation of thiol 8 by 2-chloroethanol (5). Compound 5 (2.05 g, $25.5 \mathrm{mmol})$ was dissolved in $\mathrm{H}_{2} \mathrm{O}(35 \mathrm{~mL})$ and added dropwise to a solution of thiol $8(7.71 \mathrm{~g}, 25.0 \mathrm{mmol})$ and $\mathrm{Na}_{2} \mathrm{CO}_{3}(1.98 \mathrm{~g}, 18.5 \mathrm{mmol})$ in $\mathrm{H}_{2} \mathrm{O}(35 \mathrm{~mL})$ for a period of $15 \mathrm{~min}$ at $44-39{ }^{\circ} \mathrm{C}$. The resulting solution was stirred at $39-50{ }^{\circ} \mathrm{C}$ for $9 \mathrm{~h}$, then cooled, extracted with $\mathrm{CH}_{2} \mathrm{Cl}_{2}(2 \times 10$ $\mathrm{mL}$ ) and dried $\left(\mathrm{MgSO}_{4}\right)$. The solvent was distilled off and the residue was crystallized to give product 6 (5.94 $\mathrm{g}$, $59 \%$ yield) as colorless solid, $\mathrm{mp}\left(\mathrm{CH}_{2} \mathrm{Cl}_{2}\right) 56-57^{\circ} \mathrm{C}$. IR ( $\left.\mathrm{KBr}, \mathrm{v}_{\max }, \mathrm{cm}^{-1}\right)$ : 3373(m), 2943(w), 2912(w), 2887(w), 1603(w), 1450(s), 1412(m), 1350(s), 1302(w), 1271(m), 1248(w), 1182(s), 1146(s), 1082(m), 1063(s), 1020(m), 1005(w), 955(m), 876(m), 729(w), 702(m), 683(w), 661(w), 650(w). UV, $\lambda_{\max , n m}(\log \varepsilon): 219$ (4.08), 284 (3.82). ${ }^{1} \mathrm{H} N M R\left[\left(\mathrm{CD}_{3}\right)_{2} \mathrm{CO}, 300.13 \mathrm{MHz}\right], \delta_{\mathrm{H}} 3.06\left(\mathrm{t},{ }^{3} \mathrm{JHH}_{\mathrm{HH}} 6.5,2 \mathrm{H}, \mathrm{CH}_{2} \mathrm{~S}\right), 3.22\left(\mathrm{t},{ }^{3} \mathrm{H}_{\mathrm{HH}} 6.2,2 \mathrm{H}, \mathrm{CH}_{2} \mathrm{~S}\right), 3.63-3.83(\mathrm{~m}, 4 \mathrm{H}$, $\left.2 \mathrm{CH}_{2} \mathrm{O}\right), 3.93\left(\mathrm{t},{ }^{3} \mathrm{~J}_{\mathrm{HH}} 5.6,1 \mathrm{H}, \mathrm{OH}\right), 4.05\left(\mathrm{t},{ }^{3} J_{\mathrm{HH}} 5.6,1 \mathrm{H}, \mathrm{OH}\right) .{ }^{13} \mathrm{C} \mathrm{NMR}\left(\mathrm{CD}_{3} \mathrm{CN}, 100.6 \mathrm{MHz}\right): \delta_{\mathrm{C}} 37.4\left[\mathrm{t},{ }^{4} \mathrm{CF}_{\mathrm{CF}} 3.0\right.$, $\mathrm{CH}_{2} \mathrm{~S}-(3)$ ], 39.0 [d, $\left.{ }^{4} J_{\mathrm{CF}} 4.6, \underline{\mathrm{C}} \mathrm{H}_{2} \mathrm{~S}-(1)\right], 61.8\left(\underline{\mathrm{C}} \mathrm{H}_{2} \mathrm{O}\right), 62.0\left(\underline{\mathrm{C}}_{2} \mathrm{O}\right), 119.1$ [dd, $\left.{ }^{2} J_{\mathrm{CF}} 25.1,{ }^{3} J_{\mathrm{CF}} 4.0, \underline{\mathrm{C}}-(1)\right], 120.1[\mathrm{dd}$, ${ }^{2} J_{\mathrm{CF}} 27.7,{ }^{2} J_{\mathrm{CF}} 18.7, \underline{\mathrm{C}}-(3)$ ], 121.2 [qd, $\left.{ }^{2} J_{\mathrm{CF}} 30.6,{ }^{2} J_{\mathrm{CF}} 5.9, \underline{\mathrm{C}}-(6)\right], 123.2$ (qd, ${ }^{1} J_{\mathrm{CF}} 274.8,{ }^{3} J_{\mathrm{CF}} 2.7, \underline{\mathrm{CF}} 3$ ), $146.5\left[\mathrm{ddd},{ }^{1} J_{\mathrm{CF}}\right.$ $\left.259.9,{ }^{2} J_{C F} 16.9,{ }^{4} J_{C F} 2.7, \underline{C}-(5)\right], 152.3$ [ddd, ${ }^{1} J_{C F} 248.0,{ }^{2} J_{C F} 15.4,{ }^{3} J_{C F} 6.0, \underline{C}-(4)$ ], 160.2 [dt, ${ }^{1} J_{C F} 239.9,{ }^{3} J_{C F} 3.3, \underline{C}-$ (2)]. ${ }^{19} \mathrm{~F} \mathrm{NMR}\left(\mathrm{CDCl}_{3}, 282.4 \mathrm{MHz}\right): \delta_{\mathrm{F}}-149.0\left[\mathrm{qdd},{ }^{4} J_{\mathrm{FF}} 34.6,{ }^{3} J_{\mathrm{FF}} 21.9,{ }^{5} J_{\mathrm{FF}} 13.6, \mathrm{~F}-(5)\right],-124.5\left[\mathrm{dd},{ }^{3} J_{\mathrm{FF}} 21.9,{ }^{4} J_{\mathrm{FF}}\right.$ 5.1, F-(4)], -97.5 [dd, ${ }^{5} J_{F F} 13.6,{ }^{4} J_{F F} 5.1, F-(2)$ ], -55.7 (d, ${ }^{4} J_{F F} 34.6,3 F, C F_{3}$ ). Anal. cacld. for $\mathrm{C}_{11} \mathrm{H}_{10} \mathrm{~F}_{6} \mathrm{O}_{2} \mathrm{~S}_{2}: \mathrm{C}_{37.50}$; H 2.86; F 32.36; S 18.20; $\mathrm{m} / \mathrm{z}$ 352.0021. Found: C 37.24; H 2.70; F 32.31; S 18.22; $\mathrm{m} / \mathrm{z} 352.0018$.

2,2',2"'-\{[3,6-Difluoro-5-(trifluoromethyl)benzene-1,2,4-triyl]tris(sulfanediyl)\}triethanol (7). Reaction of perfluorotoluene (1) and 2-mercaptoethanol (2) in ratio 1:3. Compound 2 (1.19 g, $15.1 \mathrm{mmol})$ was added to the solution of arene $1(1.17 \mathrm{~g}, 4.91 \mathrm{mmol})$ in DMF $(12 \mathrm{~mL})$ in the presence of $\mathrm{K}_{2} \mathrm{CO}_{3}(1.61 \mathrm{~g}, 11.5 \mathrm{mmol})$. After $8.5 \mathrm{~h}$ stirring at $20-25{ }^{\circ} \mathrm{C}$ the resulting mixture was poured into $5 \%$ aq. $\mathrm{HCl}(50 \mathrm{~mL})$. The precipitate was filtered off, dried over $\mathrm{P}_{2} \mathrm{O}_{5}$ and recrystallized to give compound 7 (1.42 g, 70\% yield). Colorless powder, $\mathrm{mp} 58-60{ }^{\circ} \mathrm{C}$ $\left(\mathrm{CH}_{2} \mathrm{Cl}_{2}\right)$. IR $\left(\mathrm{KBr}, V_{\max }, \mathrm{cm}^{-1}\right): 3522(\mathrm{~s}), 3361(\mathrm{~s}), 2941(\mathrm{~m}), 2922(\mathrm{~m}), 2883(\mathrm{~m}), 2870(\mathrm{~m}), 1610(\mathrm{w}), 1568(\mathrm{~m})$, 
1525(m), 1454(m), 1431(m), 1400(s), 1385(s), 1294(s), 1257(s), 1203(m), 1184(s), 1140(s), 1128(s), 1055(s), 1014(s), 945(w), 926(w), 860(s), 690(w), 660(m), 631(m). UV, $\lambda_{\max }, \mathrm{nm}(\log \varepsilon): 234$ (4.15), 273 (3.97), 325 (3.91). ${ }^{1} \mathrm{H}$ NMR $\left[\left(\mathrm{CD}_{3}\right)_{2} \mathrm{CO}, 400.13 \mathrm{MHz}\right]: \delta_{\mathrm{H}} 3.11\left(\mathrm{t}, 3^{3} \mathrm{JHH}_{\mathrm{HH}} 6.4,2 \mathrm{H}, \mathrm{CH}_{2} \mathrm{~S}\right), 3.14\left(\mathrm{t}, 3^{3} \mathrm{JHH}_{\mathrm{H}} 6.3,2 \mathrm{H}, \mathrm{CH}_{2} \mathrm{~S}\right), 3.26\left(\mathrm{t},{ }^{3} \mathrm{~J}_{\mathrm{HH}}\right.$ 6.1, $\left.2 \mathrm{H}, \mathrm{CH}_{2} \mathrm{~S}\right), 3.66-3.76\left(\mathrm{~m}, 6 \mathrm{H}, 3 \mathrm{CH}_{2} \mathrm{O}\right), 3.99\left(\mathrm{t},{ }^{3} \mathrm{H}_{\mathrm{HH}} 5.4,1 \mathrm{H}, \mathrm{OH}\right), 4.04\left(\mathrm{t},{ }^{3} J_{\mathrm{HH}} 5.7,1 \mathrm{H}, \mathrm{OH}\right), 4.11\left(\mathrm{t},{ }^{3} \mathrm{JHH}_{\mathrm{HH}} 5.6\right.$, $1 \mathrm{H}, \mathrm{OH}) .{ }^{13} \mathrm{C} \mathrm{NMR}\left(\mathrm{CD}_{3} \mathrm{CN}, 100.6 \mathrm{MHz}\right): \delta_{\mathrm{C}} 38.3\left(\mathrm{~d},{ }^{4} J_{\mathrm{CF}} 8.1, \underline{\left.\mathrm{CH}_{2} \mathrm{~S}\right)}, 38.7\left(\mathrm{~d},{ }^{4} J_{\mathrm{CF}} 5.3, \underline{\mathrm{CH}}_{2} \mathrm{~S}\right), 38.8\left(\mathrm{~d},{ }^{4} J_{\mathrm{CF}} 5.9, \underline{\mathrm{C}} \mathrm{H}_{2} \mathrm{~S}\right)\right.$, $61.7\left(\underline{\mathrm{CH}}_{2} \mathrm{O}\right), 61.8\left(\underline{\mathrm{C}} \mathrm{H}_{2} \mathrm{O}\right), 61.9\left(\underline{\mathrm{C}} \mathrm{H}_{2} \mathrm{O}\right), 119.9$ [qd, $\left.{ }^{2} J_{\mathrm{CF}} 30.8,{ }^{2} J_{\mathrm{CF}} 13.6, \underline{\mathrm{C}}-(5)\right], 123.5$ (qdd, ${ }^{1} J_{\mathrm{CF}} 275.1,{ }^{3} J_{\mathrm{CF}} 2.7,{ }^{4} J_{\mathrm{CF}}$ 2.7, $\left.\underline{C F}_{3}\right), 124.6\left(\mathrm{~d},{ }^{2} J_{\mathrm{CF}} 26.6, \underline{\mathrm{C}} \underline{\mathrm{Ar}}_{\mathrm{S}} \mathrm{S}\right), 129.9\left(\mathrm{dd},{ }^{2} J_{\mathrm{CF}} 22.4,{ }^{3} J_{\mathrm{CF}} 1.3, \underline{\mathrm{C}}_{\mathrm{Ar}} \mathrm{S}\right), 135.1,\left(\mathrm{~d},{ }^{2} J_{\mathrm{CF}} 22.6, \underline{\mathrm{C}} \underline{\mathrm{Ar}} \mathrm{S}\right), 157.0\left(\mathrm{~d},{ }^{1} J_{\mathrm{CF}}\right.$ 254.2, $\left.\underline{\mathrm{C}}_{\mathrm{Ar}} \mathrm{F}\right), 160.1$ (dd, $\left.{ }^{1} J_{\mathrm{CF}} 240.3,{ }^{4} J_{\mathrm{CF}} 2.6, \underline{\mathrm{C}}_{\mathrm{Ar}} \mathrm{F}\right) .{ }^{19} \mathrm{~F}$ NMR $\left(\mathrm{CD}_{3} \mathrm{CN}, 282.4 \mathrm{MHz}\right): \delta_{\mathrm{F}}-105.2\left[\mathrm{qd},{ }^{4} J_{\mathrm{FF}} 35.5,{ }^{5} J_{\mathrm{FF}} 15.1\right.$, F-(3)], -95.2 [d, ${ }^{5} J_{F F} 15.1, F-(6)$ ], $-53.0\left(d,{ }^{4} J_{F F} 35.5,3 F, C_{3}\right)$. Anal. cacld for $\mathrm{C}_{13} \mathrm{H}_{15} \mathrm{~F}_{5} \mathrm{O}_{3} \mathrm{~S}_{3}$ : C 38.04; $\mathrm{H} 3.44 ; \mathrm{F}$ 23.14; S 23.43; $m / z$ 410.0098. Found: C 38.07; H 3.44; F 23.11; S 23.58; $m / z 410.0100$.

\section{Acknowledgements}

The authors thank the Multi-Access Chemical Research Center of the Siberian Branch of the Russian Academy of Sciences for analytic measurements. The work was financially supported by Russian Science Foundation (grant 16-13-10156)

\section{References}

1. Wu, W.; Qin, J.; Li, Z. Polymer 2013, 54, 4351-4382.

http://dx.doi.org/10.1016/i.polymer.2013.05.039.

2. Abd-El-Aziz, A. S.; Strohm, E. A. Polymer 2012, 53, 4879-4921.

http://dx.doi.org/10.1016/i.polymer.2012.08.024.

3. Lee, K.-S.; Kim, R. H.; Yang, D.-Y.; Park, S. H. Prog. Polym. Sci. 2008, 33, 631-681. http://dx.doi.org/10.1016/i.progpolymsci.2008.01.001.

4. Li, Z.; Wu, W.; Hu, P.; Wu, X.; Yu, G.; Liu, Y.; Ye, C.; Li, Z.; Qin, J. Dyes Pigm. 2009, 81, $264-272$. http://dx.doi.org/10.1016/i.dyepig.2008.10.014.

5. Alijoumaa, K.; Qi, Y.; Ding, J.; Delaire, J. A. Macromolecules, 2009, 42, 9275-9288. http://dx.doi.org/10.1021/ma900930q.

6. Wu, W.; Huang, Q.; Zhong, C.; Ye, C.; Qin, J.; Li, Z. Polymer 2013, 54, 5655-5664. http://dx.doi.org/10.1016/i.polymer.2013.07.073.

7. Strehmel, B.; Sarker, A. M.; Detert, H. ChemPhysChem. 2003, 4, 249-259. https://doi.org/10.1002/cphc.200390041.

8. Siao, Y.-Y.; Shau, S.-M.; Hu, S.-H.; Lee, R.-H.; Lin, C.-H.; Wu, J.-Y.; Jeng, R.-J.Polymer 2013, 54, 3850-3859. http://dx.doi.org/10.1016/i.polymer.2013.05.040.

9. Sapegin, A.; Krasavin, M. J. Fluorine Chem. 2018, 209, 56-64. http://dx.doi.org/10.1016/i.jfluchem.2018.02.002.

10. Facchetti, A.; Yoon, M.-H.; Stern, C. L.; Katz, H. E.; Marks, T. J. Angew. Chem. Int. Ed. 2003, 42, 3900-3903. http://dx.doi.org/10.1002/anie.200351253.

11. Winkler, B.; Meghdadi, F.; Tasch, S.; Müllner, R.; Resel, R.; Saf, R.; Leising, G.; Stelzer, F. Opt. Mater. (Amsterdam, Neth.) 1998, 9, 159-162. 
12. Tkachenko, I. M.; Belov, N. A.; Kobzar, Y. L.; Dorokhin, A. V.; Shekera, O. V.; Shantarovich, V. P.; Bekeshev, V. G.; Shevchenko, V. V. J. Fluorine Chem. 2017, 195, 1-12.

http://dx.doi.org/10.1016/i.jfluchem.2017.01.008.

13. Park, N. H.; Gomes, G. d. P.; Fevre, M.; Jones, G. O.; Alabugin, I. V.; Hedrick, J. L. Nature Commun. 2017, 8, Article number: 1553.

http://dx.doi.org/10.1038/s41467-017-00186-3.

14. Langner, C.; Meier-Haack, J.; Voit, B.; Komber, H. J. Fluorine Chem. 2013, 156, 314-321. http://dx.doi.org/10.1016/i.jfluchem.2013.07.013.

15. Amosova, S. V.; Gavrilova, G. M.; Afonin, A. V. Russ. J. Org. Chem. 2005, 41, 402-405. https://doi.org/10.1007/s11178-005-0178-y.

16. Hynes, R. C.; Peach, M. E. J. Fluorine Chem. 1986, 31, 129-133.

https://doi.org/10.1016/S0022-1139(00)80528-X.

17. Brooke, G. M. J. Fluorine Chem. 1997, 86, 1-76, and references therein. http://dx.doi.org/10.1016/S0022-1139(97)00006-7

18. Kalhor-Monfared, S.; Jafari, M. R.; Patterson, J. T.; Kitov, P. I.; Dwyer, J. J.; Nuss, J. M.; Derda, R. Chem. Sci. 2016, 7, 3785-3790 and correction Chem. Sci. 2017, 8, 807. http://dx.doi.org/10.1039/c5sc03856a http://dx.doi.org/10.1039/c6sc90071b

19. Niembo, S.; Vallribera, A.; Moreno-Manas, M. New J. Chem. 2008, 32, 94-98. http://dx.doi.org/10.1039/b707776a

20. Umemoto, T.; Garrick, L. M.; Saito, N. Beil. J. Org. Chem. 2012, 8, 461-471. http://dx.doi.org/10.3762/bjoc.8.53

21. Kizner, T. A.; Shteingarts, V. D. Zh. Org. Khim. 1989, 25, 2424-2431. Chem. Abstr. 1990, 112, 25, $234891 z$.

22. Petrucci, R. H., Herring, F. G., Madura, J. D., Bissonnette, C. General Chemistry: Principles and Modern Applications. 10 $0^{\text {th }}$ ed. Pearson Canada Inc., Toronto, Ontario, 2011.

23. Fadeeva, V. P.; Tikhova, V. D.; Nikulicheva, O. N. J. Anal. Chem. 2008, 63, 1094-1106. https://doi.org/10.1134/S1061934808110142

24. Maksimov, A. M.; Platonov, V. E. Fluorine Notes 1999, 4, 5-6. http://notes.fluorine1.ru/contents/history/1999/4 1999/letters/index.html 